\title{
Reflets
}

Revue d'intervention sociale et communautaire

\section{De la revendication au partenariat : la Société franco-manitobaine au coeur du développement économique des francophones du Manitoba}

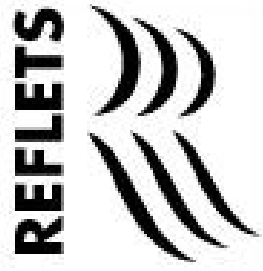

\section{Halimatou BA}

Volume 18, numéro 1, printemps 2012

URI : https://id.erudit.org/iderudit/1012335ar

DOI : https://doi.org/10.7202/1012335ar

Aller au sommaire du numéro

Éditeur(s)

Reflets, Revue d'intervention sociale et communautaire

ISSN

1203-4576 (imprimé)

1712-8498 (numérique)

Découvrir la revue

Citer cet article

BA, H. (2012). De la revendication au partenariat : la Société

franco-manitobaine au coeur du développement économique des

francophones du Manitoba. Reflets, 18(1), 140-154.

https://doi.org/10.7202/1012335ar d'utilisation que vous pouvez consulter en ligne. 


\section{De la revendication au partenariat : la Société franco-manitobaine au cœur du développement économique des francophones du Manitoba}

Halimatou BA, Ph. D.

Professeure adjointe en travail social, Université de Saint-Boniface

\section{Introduction}

Les années passent et marquent d'importants changements dans le profil des communautés francophones du Manitoba. Sur le plan démographique, celles-ci ont connu des hauts et des bas, allant de population largement majoritaire, il y a plus d'un siècle, à une minorité qui représente en 2006 à peine plus de $4 \%$ de la population totale de la province. La faiblesse de leur poids démographique n'a pas pour autant freiné le dynamisme des Franco-manitobains dans leurs efforts pour conserver leur langue, participer au rayonnement des activités culturelles et vouloir imposer leur influence économique et politique (Allain, 2002). Il est possible de se faire servir en français dans un restaurant à Winnipeg, de participer à un groupe pour femmes francophones violentées ou même de diriger en français le conseil d'administration d'un organisme communautaire.

Pour en arriver là, il a fallu que les francophones passent par un long parcours parsemé d'embuches, de rebondissements, de 
victoires et de déceptions (Rocque, 2011). À différents moments de leur histoire, les Franco-manitobains ont dû mener des actions collectives de revendication auprès des autorités en place ou de collaboration avec elles. Ils ont réalisé un travail de construction sociale et identitaire qui constitue un exemple édifiant de résistance et d'affirmation collective par comparaison à d'autres communautés francophones en semblable situation (Allain, 2002).

Ces changements n'ont pas pour autant fait disparaître les nombreuses difficultés que vivent les communautés en situation minoritaire : survivance linguistique, problèmes d'intégration sociale, pauvreté, violence, assimilation, problèmes de santé et absence de certains services sociaux essentiels en français. Fiers de recouvrer certains de leurs droits au sein d'une société majoritairement anglophone, les francophones du Manitoba peuvent maintenant entreprendre un partenariat institutionnel avec les autorités en place et promouvoir un développement économique de leurs communautés afin de s'y épanouir.

Une de ces collaborations se réalise sous l'égide de la Société franco-manitobaine (SFM), la structure organisationnelle étendard créée dans les années 1960 et qui chapeaute les actions menées par trente-six organismes communautaires. Le nouveau contexte a contribué au renforcement de la mission de la SFM qui est de guider et d'aider les communautés à réclamer leurs droits, à sauvegarder leurs acquis historiques, culturels et identitaires et à relever les multiples défis économiques.

Dans cet article, nous esquissons un portrait des communautés minoritaires francophones du Manitoba. Nous situons la SFM comme acteur social de leur développement à travers les objectifs d'intervention définis dans son plan global. Ces objectifs ciblent les principales problématiques qui confrontent les populations francophones et francophiles. Puis, nous nous penchons sur l'apport de l'immigration francophone. Pour conclure, nous identifions quelques défis à relever pour le développement des communautés francophones du Manitoba ${ }^{1}$. 


\section{Qui sont les francophones du Manitoba?}

Quand on parle du Manitoba, on doit se rappeler que cette province a été fondée par les francophones. Les communautés francophones étaient majoritaires bien avant l'adhésion de la province à la Confédération canadienne en 1870 (Marchand, 2004; Sing, 2005). Classés en troisième position après les Allemands et les Anglais, les francophones ont subi un déclin et sont devenus minoritaires (Statistique Canada, 2002). Le recensement de 2006 mentionne que 44110 habitants du Manitoba parlent le français comme première langue. Ils représentent maintenant $4 \%$ des 1133515 habitants qui constituent la population totale de la province (Statistique Canada, 2006).

La communauté francophone est essentiellement urbaine ou semi-urbaine. Près de $80 \%$ de cette population vit à Winnipeg ou dans ses alentours. La population francophone qui réside à Winnipeg a tendance à se concentrer dans les quartiers historiquement francophones comme Saint-Boniface, Saint-Vital et Saint-Norbert (de Moissac et collab., 2011). Cette communauté forme une mosaïque linguistique et identitaire. Si bien, qu'il est plus convenable de parler de francophonies manitobaines au pluriel (Marchand, 2004). Cette diversité des populations francophones dans les communautés respectives urbaines et rurales peut être classée en trois catégories : les francophones de souche, les francophiles ou franglophones et les nouveaux immigrants francophones.

Implantés depuis longtemps, les francophones de souche ont pour la plupart leurs racines au Québec. Les autres sont des Métis ou encore, des immigrants venus d'Europe. L'histoire du Manitoba est en effet intimement liée à celle de l'immigration et les premiers immigrants européens à s'aventurer dans l'ouest du Canada étaient des francophones. Le commerce des fourrures avec les Premières nations a permis leur établissement définitif et la formation d'une nation métis (Marchand, 2004). Celle-ci restera marginalisée des autres populations et distante de ses origines françaises. En dehors des francophones de Saint-Boniface, 
d'autres Métis francophones habitent plusieurs autres villages du Manitoba. Les populations dans la région de la Montagne sont aussi des descendants d'immigrants français et belges qui sont venus s'y établir à la fin du XIX ${ }^{\mathrm{e}}$ et au début du XX $\mathrm{XX}^{\mathrm{e}}$ siècle. Ce fait témoigne dès le départ de l'existence d'une diversité francophone (Jourdain, 2011).

Les francophiles ou franglophones qui parlent le français constituent cette catégorie de francophones qui ont appris la langue dans les écoles d'immersion ou autrement. L'histoire rappelle que vers la fin du XIX ${ }^{\mathrm{e}}$ siècle, avec l'arrivée du chemin de fer et l'échec du recrutement des Français venant de l'est du Canada, la population francophone a décliné pour devenir très minoritaire au profit de l'immigration ontarienne et britannique. Le phénomène des mariages mixtes s'est développé et a permis aux enfants qui en sont issus de se sentir à la fois francophones et anglophones. Ces derniers faisant partie des communautés francophones peuvent désormais se targuer d'avoir une identité multidimensionnelle. L'immigration anglophone au Manitoba n'a pas été favorable à la francophonie, ni à l'éducation des francophones. Les immigrants étaient composés en majorité d'anglophones, d'Allemands, d'Islandais, de Philippins, d'Ukrainiens, qui venaient tous grossir les rangs de la population anglophone, au grand dam des francophones qui voyaient décliner leur poids démographique. Cette baisse a conduit en partie à l'assimilation des communautés francophones en les rendant plus perméables aux influences de l'extérieur. L'Église a joué un rôle important dans le devenir de la francophonie dans les Prairies, la majorité de la population étant constituée à l'origine de Métis qui sont catholiques et d'expression française (Jourdain, 2011). Il faut dire que le Official Language Act de 1890 qui interdisait l'enseignement du français dans les écoles publiques pendant près de quatre-vingt-dix ans n'a pas aidé la cause des francophones.

La nouvelle génération de francophones s'est beaucoup développée à partir des années 1990. En plus des francophones venant de l'est du Canada et de l'Europe, les nouveaux immigrants francophones à s'installer au Manitoba viennent majoritairement de l'Afrique francophone. Ils augmentent la communauté par leur nombre et 
par la diversité de leurs origines. On ne peut plus désormais parler uniquement de Franco-manitobains, mais de francophones du Manitoba. La réflexion de ce répondant en témoigne :

"Je me souviens, quand j'avais commencé à enseigner au collège universitaire de Saint-Boniface - devenu Université de Saint Boniface - au milieu des années 1980. La population était très homogène, canadienne-française, composée de Franco-manitobains, de Québécois et d'Acadiens, entre autres. Mais ce que l'on voit maintenant à l'USB, c'est beaucoup une plus grande diversité, avec un grand nombre d'immigrants dont la plupart viennent d'Afrique."

Il est évident que l'immigration joue un rôle décisif dans la croissance de la population canadienne. Elle a permis un développement rapide de celle du Manitoba. Elle est cependant majoritairement anglophone. Il devient donc crucial, dans le recrutement des immigrants, de permettre à la communauté francophone de s'accroître proportionnellement à celle des anglophones. Les francophones du Manitoba veillent à ce que cette proportion soit respectée. Les nouvelles ouvertures politiques en matière d'immigration le permettent. En effet, selon ce répondant :

«Avant, le Manitoba ne recevait pas plus de 30 immigrants francophones par année. C'est à partir de 1990 que les choses ont beaucoup changé. Maintenant, on peut avoir de 200 à 300 immigrants francophones par année.»

\section{Le rôle de la Société franco-manitobaine dans les changements au sein des communautés francophones}

Historiquement, la situation des communautés francophones était très préoccupante, mais en même temps étonnante. Les 
francophones étaient déterminés à "survivre ", même en situation minoritaire, et même dans des circonstances peu favorables. Privées d'écoles pendant plus d'un demi-siècle, les communautés francophones ont subi des lois assimilatoires et l'anglais est devenu la seule langue officielle du Manitoba. Leurs droits étant bafoués, les communautés francophones ont réussi à conserver leur langue et leur culture en vivant dans des communautés homogènes, restées catholiques et isolées. À l'heure actuelle, la situation semble s'améliorer avec la présence d'une politique linguistique favorable au bilinguisme. En effet, à la fin des années 1960, le gouvernement fédéral intervient pour reconnaître le caractère bilingue de l'ensemble du pays et appuyer la revitalisation des collectivités francophones minoritaires à l'échelle nationale. La Loi sur les langues officielles de 1969 reconnaissait le principe du bilinguisme officiel au niveau de l'état fédéral et obligeait, pour la première fois, le gouvernement fédéral à fournir des services dans les deux langues officielles. Plus tard, en 1982, pour favoriser davantage la protection et l'épanouissement de la minorité francophone, la Charte canadienne des droits et libertés a accordé le droit à l'éducation en français dans l'ensemble du Canada (Jourdain, 2011). On peut dire que l'adoption de cette loi avec ses éléments relatifs aux droits collectifs des minorités est une grande victoire. Elle a permis la création des écoles françaises et de tous les mécanismes qui facilitent leur gestion.

Pour obtenir ces gains et voir à leurs besoins, il a fallu sur le plan politique que les communautés francophones du Manitoba se regroupent pour bâtir un important réseau d'institutions qui œuvrent pour la sauvegarde de leurs droits, l'instauration de changements dans leurs conditions de vie et la résolution de problèmes sociaux découlant de leur statut minoritaire. À la suite d'une longue et minutieuse consultation auprès de l'ensemble des communautés, un plan global a été élaboré dans le cadre du renouvellement de l'entente Canada-communauté francomanitobaine (Allaire, 1999). Une des stratégies développées a été de créer à la fin des années 1960 une structure officielle qui sert de cadre de référence pour toute la communauté : la Société francomanitobaine (SFM). Lors de sa création, cette dernière était d'abord 
et avant tout une structure de revendications pour la restauration des droits des Franco-manitobains. À l'heure actuelle, elle est devenue l'organe politique, le porte-parole officiel des membres de la communauté francophone sur les scènes provinciale et fédérale. À travers un grand projet de développement communautaire, Agrandir l'espace francophone adopté en 2001, la SFM se donne les moyens de réaliser sa mission de coopération entre les différentes communautés francophones au Manitoba, celle d'assurer la pleine vitalité et un épanouissement de tous les secteurs d'activités et auprès de toutes les clientèles francophones et de tous les âges. En cela, elle collabore étroitement avec les autorités fédérales et provinciales. Cependant, certains besoins se font toujours sentir et retiennent l'attention des acteurs et intervenants sociaux. Il y a quelques années, la SFM a eu l'occasion de mettre en œuvre un autre projet, Changement 2008, qui a permis aux organismes communautaires franco-manitobains de se concerter et d'établir un modèle de collaboration.

Sur la base de ces initiatives, plusieurs réalisations ont été menées à bien et des progrès considérables sont constatés dans plusieurs domaines. Le Manitoba francophone a obtenu des gains importants et la collectivité francophone bénéficie de nombreuses infrastructures dont elle a besoin pour vivre et s'épanouir dans sa langue. Entre autres gains importants, nous pouvons citer la création du Secrétariat aux affaires francophones qui sert de conseiller auprès du gouvernement du Manitoba, le Collège universitaire de Saint-Boniface devenu depuis septembre 2011 l'Université de Saint-Boniface, l'université francophone la plus à l'ouest du Canada, la révision de la politique des services en langue française en 1999, le Conseil communauté en santé du Manitoba implanté en 2004, le Consortium national de formation en santé (de Moissac et collab., 2011). Le soutien institutionnel du gouvernement du Manitoba est nettement visible dans l'investissement des sommes substantielles pour la construction du nouveau théâtre, le Cercle Molière, et tout récemment la construction du Pavillon de la santé et des services sociaux à l'Université de Saint-Boniface. 
Le partenariat ainsi développé par la SFM avec le gouvernement provincial a favorisé le développement d'une politique de mise en place des services en français depuis une vingtaine d'années. Avec la volonté et l'appui de l'actuel premier ministre qui, de surcroît, est le député du quartier francophone de SaintBoniface, le nouveau contexte contribue au développement de la mission de la SFM et à relever les multiples défis à venir. De plus en plus de mesures visent à appuyer l'épanouissement des francophones et à sceller définitivement une forte implication du gouvernement.

Dans son fonctionnement, la SFM chapeaute trente-six organismes communautaires qui œuvrent au bien-être et à la promotion de la francophonie et des communautés francophones avec chacun un mandat sectoriel dans différents domaines. C'est la SFM qui négocie avec le gouvernement fédéral pour l'obtention et le renouvellement de leur financement. Ce réseau communautaire s'étend aussi en dehors de la ville deWinnipeg et englobe plus de quarante villages qui ceinturent Winnipeg. La SFM a été visionnaire dans la mise sur pied de son plan stratégique d'ensemble, lequel vise à assurer l'épanouissement et la vitalité de la communauté francophone. Il comporte cinq volets :éducation et formation; espace francophone; santé et services sociaux; structure communautaire; vie culturelle (SFM, 2010). Il s'agit pour la SFM de promouvoir et de rendre accessible l'éducation à tous les francophones en misant sur la petite enfance, sur la construction identitaire et sur la fierté des francophones du Manitoba. Il œuvre pour l'agrandissement de l'espace francophone et pour la facilité de l'accès à des services de santé et à des services sociaux en français pour les personnes qui souhaitent être servies dans leur langue maternelle. Il tente un virage des francophones vers le développement économique de leurs communautés, en misant sur un développement local qui permet aux francophones de se prendre en charge eux-mêmes, de mettre en place des programmes sociaux pour et par eux et de favoriser le réseautage et la collaboration. Un répondant est fier des initiatives prises en ce sens :

«[...] une mission de l'ANIM [Agence bilingue d'échanges commerciaux du Manitoba] s'est 
rendue au Mali pour développer des possibilités de coopération avec des municipalités. En octobre 2010, l'ANIM a organisé un symposium appelé «Centralia » qui a regroupé plus de 400 participants à travers le monde. Ce symposium était bilingue et les francophones et anglophones participaient à qualité égale. C'était une première dans l'histoire du Manitoba de voir des francophones traiter de questions de finances, d'affaires et de faire du réseautage. Le but est d'ouvrir le Manitoba à des marchés francophones extérieurs, à commencer par le Québec, mais aussi à ouvrir les marchés extérieurs aux entreprises internes du Manitoba. Une deuxième édition est prévue pour 2012!»

\section{L'apport de l'immigration francophone}

Les francophones ont toujours été solidaires. Cela leur a permis de survivre. Le constat est que cette population francophone ne se renouvelle pas au même rythme que celle anglophone. Elle demeure fortement adulte avec un taux de $21 \%$ de personnes âgées de plus de 65 ans (de Moissac et collab., 2011). Les francophones de "souche " ont lutté pour obtenir des droits. Pour continuer de se développer et de s'enrichir, ils ont maintenant besoin de personnes avec qui partager ces acquis. Ils ont tout intérêt à s'ouvrir à d'autres communautés pour se maintenir, développer et enrichir leur patrimoine identitaire et culturel. Sur ce plan, on peut dire que la francophonie manitobaine se diversifie depuis une vingtaine d'années. Cette vision d'ouverture au monde francophone a d'ailleurs propulsé des changements majeurs à partir des années 1990. Ils se sont développés davantage en 2005 et 2006. Et dès 2007, le Manitoba a accueilli 334 immigrants capables de s'exprimer en français, ce qui représente une hausse de $27 \%$ par rapport à l'année précédente. Cette progression découle des initiatives de marketing et de recrutement menées en France, 
en Belgique et en Afrique francophone (Ka, 2007). Ce même programme prévoit la venue de 10000 immigrants par année, $7 \%$ d'entre eux étant destinés à la communauté francophone. Cette cible de 700 immigrants francophones par année représente donc un atout majeur pour la communauté. L'Université de SaintBoniface a aussi joué un rôle essentiel dans la recherche d'étudiants internationaux. À cet effet, il faut soutenir le rayonnement de l'université en renforçant son pouvoir institutionnel dans l'espace francophone à l'ouest du Canada. La création de l'École de service social en français découle de cet effort. Il y a une insuffisance de travailleurs sociaux pour les francophones et le besoin risque d'augmenter avec l'arrivée massive de personnes immigrantes. D'où l'importance d'avoir des mesures d'accompagnement pour faire face à ce développement démographique.

L'Accueil francophone est l'un des 36 organismes affiliés à la SFM. Sa mission est de soutenir les nouveaux arrivants de langue française. Il lui incombe de présenter une image favorable de la francophonie au Manitoba (Dalley, 2003). Créé en 2003, l'Accueil francophone s'est considérablement développé dans la prise en charge des immigrants reçus et des réfugiés francophones. Il leur offre une gamme très variée de services, qui vont de l'accueil à l'aéroport jusqu'à leur établissement dans la communauté, et ce, sur pour période de deux ans. Ces services touchent la sensibilisation, le logement, le jumelage avec une famille d'accueil, le tutorat et le mentorat, la traduction et l'interprétation, le notariat et l'assermentation. On y fait aussi de l'accompagnement et de la résolution en situation de crise. L'Accueil francophone agit aussi comme organisme de transition entre les immigrants et les autres organismes auxquels ils pourraient avoir recours. En ce sens il fait beaucoup de références dans ses interventions. Un informateurclé se prononce sur son impact :

"L'Immigration a joué un rôle capital dans les changements survenus au Manitoba, dans le sens où les immigrants viennent donner un souffle nouveau à la communauté francophone. [...] Ces gens-là assument leadership au sein de la communauté; et aussi nous aident à construire 
une masse critique pour l'obtention de services

de toutes sortes auprès du gouvernement. "

\section{Enjeux et défis du développement de la francophonie manitobaine}

Depuis sa création, la SFM a mené des actions collectives qui portent aujourd'hui des fruits.

La communauté francophone a beaucoup évolué passant du stade de survie, dans les années 1960-1970, à celui de pleine vie vers les années 1990. Les francophones ont atteint une certaine stabilité; il leur faut œuvrer pour la maintenir et passer à un stade d'épanouissement. En amorçant ce virage, la SFM mise sur la participation et le partenariat pour promouvoir le développement économique des communautés. Elle fait en amont tout le travail de revendication, de collaboration et de partenariat pour obtenir le plus grand nombre de services gouvernementaux. En aval, elle mène des démarches de concertation pour coordonner tout le réseau d'organismes communautaires qu'elle chapeaute pour une meilleure gouvernance et établit des passerelles avec la communauté anglophone. Pour en arriver là, elle a déployé à différents moments de sa lutte des stratégies, d'abord conflictuelles, puis consensuelles et enfin, partenariales. La SFM a facilité la communication avec les autorités en place et a contribué pleinement au développement de l'espace francophone. Sur ce plan, la création des écoles francophones, les différents programmes de recrutement d'immigrants, le plan stratégique, le développement de l'Université de Saint-Boniface et la création de l'Ecole de service social pour la formation en français des travailleurs sociaux sont autant d'éléments favorables pour la réalisation de cet objectif.

Cependant, de nombreux défis restent à surmonter, parmi lesquels figure le maintien de la croissance démographique francophone pour ne pas perdre son importance déjà restreinte dans un milieu anglophone. De plus en plus d'immigrants francophones choisissent la destination Manitoba. La rétention de ces derniers 
dépend de leur capacité à s'intégrer dans leur nouveau milieu et des opportunités économiques, sociales et politiques qui s'offrent à eux. Sans exclure le risque de ghettoïsation pour ces immigrants, le développement et le maintien du français nécessiteront une concentration géographique des communautés là où elles peuvent avoir facilement accès à des services en français. Or, à Winnipeg par exemple, le manque de logements adéquats, disponibles et accessibles demeure un grand problème et pousse les francophones à habiter hors des quartiers francophones habituels comme SaintBoniface (Alper et collab., 2011). Il faut davantage prendre des mesures d'accompagnement pour les intégrer tous et leur donner l'occasion de prouver que leur présence est aussi nécessaire que valorisante pour la province. Cette présence permet d'attirer les entreprises et de leur fournir une main-d'œuvre bilingue. Des institutions de formation existent et c'est très important de faire progresser la francophonie dans différents secteurs de la vie, tels que la santé, l'éducation ou la fonction publique.

Un autre défi de la SFM consiste à combattre la perte d'identité de francophones dont la langue maternelle s'émousse de jour en jour. Ce combat passe par la réduction de l'avancée de l'assimilation et la conservation des droits acquis. Pour ce faire, il semble nécessaire de développer les services en français qui existent déjà et d'en promouvoir de nouveaux. Il n'y a pas d'équilibre dans l'offre des services lorsque ceux-ci sont effectués en anglais ou en français. Le profil de la francophonie manitobaine esquissé ci-dessus porte à méditer sur le vocable " minoritaire " qui peut être compris différemment. D'un côté, on peut ne pas considérer comme minoritaire un francophone de souche, car il est caucasien. De l'autre, un francophone d'origine ethnoculturelle peut appartenir à une double ou triple minorité de par sa terre natale, la couleur de sa peau et même son appartenance religieuse. Ces deux catégories de francophones subissent différemment l'impact des barrières linguistiques dans leur communauté naturelle. Dans ces communautés, la plupart des francophones de souche travaillent en anglais et sont habituellement bilingues. Quand ils demandent un service en français, c'est une question de préférence personnelle. Et si le service leur est offert en anglais, ils peuvent aussi s'en 
accommoder. La situation differe pour les personnes immigrantes, surtout d'origine africaine, qui n'ont que le français comme langue du pays d'accueil. Elles doivent maîtriser l'anglais pour trouver de l'emploi. Le cas particulier des réfugiés ayant de grands besoins sur le plan de la santé et de l'adaptation sociale est préoccupant. Ils sont plus susceptibles de faire appel à un plus grand nombre de services gouvernementaux, largement anglophones, que le citoyen moyen. Par ailleurs, dans les villages francophones traditionnels comme Saint-Malo, Saint-Pierre-Jolys, Saint-Jean-Baptiste ou Notre-Dame-de-Lourdes, il y a des citoyens francophones âgés qui se débrouillent en anglais, mais qui se sentent plus à l'aise en français lorsqu'ils ont besoin de services en santé.

Voter une loi sur les services en français favoriserait le bilinguisme et le développement de la francophonie au Manitoba. Il y a bien une politique claire concernant la francophonie, mais une loi aurait plus de force qu'une politique. La présence d'un Secrétariat aux affaires francophones est une initiative gouvernementale appréciée. Cependant, l'adoption d'une loi constitue une priorité. Celle-ci garantirait les acquis et ouvrirait d'autres opportunités aux francophones. En attendant, sur le plan politique, il faut encourager la création de postes bilingues dans les institutions provinciales et fédérales et veiller à ce qu'ils soient occupés par des personnes qui sont parfaitement bilingues. Le Manitoba pourrait espérer devenir un jour une province officiellement bilingue, occasion qu'il a ratée lors de sa création à cause de difficultés conjoncturelles et de déchirements sur la question linguistique (Marchand, 2004). Cependant, peu nombreux sur le plan démographique, les francophones font qualitativement toute une différence dans l'édification et le développement social de la province.

La SFM doit continuer à revendiquer le plein respect des droits garantis aux francophones ainsi que l'adoption de nouvelles lois et de nouvelles politiques gouvernementales visant l'épanouissement du français au Manitoba. Cependant, une trop grande modération lui est reprochée dans ses façons de s'affirmer publiquement, lesquelles frôleraient l'abdication à ses responsabilités (Hébert, 2011). Tout en poursuivant un travail approprié, la SFM doit faire usage de stratégies efficaces dans ses revendications politiques 
afin d'éviter les controverses publiques autour des questions francophones.

Pour conclure, nous dirons que les francophones du Manitoba tout en étant minoritaires dans une province fortement anglophone, sont déterminés à protéger leurs acquis et à promouvoir leur développement économique. Des avancées très positives ont été constatées dans leur cheminement. L'ensemble de ces progrès permet d'envisager l'avenir avec un certain optimisme. Ces dernières années, les francophones du Manitoba ont démontré leur capacité à s'ouvrir à d'autres cultures et à accueillir d'autres personnes. Le rôle de soutien et de coordination de la SFM demeure pertinent et indispensable pour bâtir des relations et les nourrir. Devenant de plus en plus diversifiés sur le plan ethnique et culturel, les francophones du Manitoba ont intérêt à rester solidaires et dynamiques. Actuellement, leur avenir repose sur des prémisses assez fragiles parce que tributaire du soutien gouvernemental et d'une solidarité entre les francophones de tout le pays, à travers la Fédération des communautés francophones et acadienne du Canada (FCFA, 2011). Sauf pour la ColombieBritannique où la réalité est différente, il semble y avoir beaucoup de ressemblances dans ce que vivent les francophones de chacune des provinces de l'Ouest. Pour développer la francophonie au Manitoba, il faut travailler dans tous les domaines. De ce point de vue, les communautés francophones sont privilégiées et bien organisées autour de la SFM. Ce qui n'est pas le cas partout.

\section{Note}

Cet article a été développé en faisant une analyse et une synthèse de données qui proviennent de deux sources de connaissances. D'abord, j'ai fait de la recherche documentaire sur le sujet en consultant quelques écrits scientifiques sur la francophonie au Manitoba et d'autres écrits réalisés sur des communautés minoritaires semblables. Par la suite, j’ai triangulé ces éléments en interrogeant quelques intervenants sociaux œuvrant dans la communauté francophone à Winnipeg. Pour ce faire, j'ai discuté avec trois personnalités bien connues dans le milieu social, politique et institutionnel à Winnipeg. Ces personnes ont une expertise certaine par rapport aux thèmes traités dans l'article. Leur position professionnelle dans la francophonie manitobaine et les responsabilités qu'elles assument font d'elles des informatrices clés typiques. Leurs propos ont été recueillis lors d'entrevues semi-dirigées, transcrites, puis analysées selon la méthode de l'analyse du contenu (L'Écuyer, 1990). Les informations condensées à partir de ces deux sources 
de données ont servi globalement à rédiger l'article qui, avec les limites qu'il peut contenir, n'a pas l'ambition de dresser un portrait exhaustif de la situation des francophones du Manitoba.

\section{Bibliographie}

ALLAIN, Greg (2002). « La communauté francophone de Saint-Jean, Nouveau-Brunswick : de la survivance à l'affirmation ", Francophonies d'Amérique, No 14, 2002, p. 37-53.

ALLAIRE, Gratien (1999). La francophonie canadienne. Portraits, CIDEF-AFI, Québec.

ALPER, David, et collab. (2011). "Les immigrants face au logement à Winnipeg : Cas des nouveaux arrivants d'Afrique francophone ", réf. du 27 février 2012, Canadian Centre for Policy Alternatives - Manitoba. http://www.policyalternatives.ca/sites/default/files/uploads/ publications/2012/01/Les\%20immigrants\%20logement\%20Alper.pdf

DALLEY, Phyllis (2003). "Définir l'accueil : enjeu pour l'immigration en milieu minoritaire francophone en Alberta ", Francophonies d'Amérique, № 16, 2003, p. 67-78.

de MOISSAC, Danielle, et collab. (2011). Disponibilité et accessibilité des services de santé en français au Manitoba : Rapport final, réf. du 27 février 2012, Université de Saint-Boniface / Institut francoontarien de l'Université Laurentienne. http://www.cusb.ca/admin/document.doc?id=442

FCFA, (Fédération des communautés francophones et acadienne du Canada). Profil de la communauté francophone du Manitoba, réf. du 12 août 2011, www.fcfc.ca/profils

HÉBERT, Raymond (2011). La SFM a manqué le bateau. Article dans le journal la Liberté, réf. du 20 novembre 2011, http://la-liberte.mb.ca/blogue-du-journal/la-sfm-a-manque-lebateau\#comment-383

JOURDAIN, Guy (2011). "La francophonie de l'Ouest canadien : regard historique ", dans Jules Rocque (dir.), La direction d'école et le leadership pédagogique en milieu francophone minoritaire considérations théoriques pour une pratique éclairée. Saint-Boniface, PUSB, p. 11-28.

KA, Mamadou (2007). «L'immigration francophone au Manitoba, 2000-2006 : un profil statistique », Cahiers franco-canadiens de l'Ouest, $\mathrm{Vol} .19, \mathrm{~N}^{\circ}$ 2, p. 119-139.

MARCHAND, Anne-Sophie (2004). "La francophonie plurielle au Manitoba ». Francophonies d'Amérique, No17, p. 147-159.

SING, Paméla V. (2005). "L'expérience du minoritaire francophone : littérature, théorie et nouvelle représentation de la différence ", Cahiers franco-canadiens de l'Ouest, Vol.17, Nos1-2, p. 3-15.

ROCQUE, Jules (2011). "La francophonie de l'Ouest canadien : aperçu démographique ». Dans Jules Rocque (dir.), La direction d'école et le leadership pédagogique en milieu francophone minoritaireconsidérations théoriques pour une pratique éclairé. Saint-Boniface, PUSB, p. 71-98

SOCIÉTÉ FRANCO-MANITOBAINE (2010). "Plan stratégique communautaire 20102015 ", réf. du 30 juin 2010, http://www.sfm.mb.ca/communaute/plan_strategique_ communautaire_2010-2015/

STATISTIQUE CANADA (2002). "Profils des langues au Canada : l'anglais, le français et bien d'autres langues ", Ottawa, ministère de l'Industrie.

STATISTIQUE CANADA (2006). «Profil linguistiques des professionnel.le.s de la santé - Tableau personnalisé du recensement de 2006 produit pour Patrimoine canadien », Ottawa, Statistique Canada. 